OPEN ACCESS

Edited by:

Yan Huang,

Harvard Medical School,

United States

Reviewed by:

Weihua Guan,

University of Minnesota Twin Cities,

United States

Zhi Liu,

University of Macau, China

*Correspondence:

Shuilin Jin

jins/@hit.edu.cn

${ }^{t}$ These authors have contributed equally to this work

Specialty section:

This article was submitted to Neurogenomics,

a section of the journal

Frontiers in Neuroscience

Received: 14 September 2018

Accepted: 18 December 2018

Published: 17 January 2019

Citation:

Wang N, Zhang Y, Xu L and Jin S

(2019) Relationship Between

Alzheimer's Disease and the Immune

System: A Meta-Analysis

of Differentially Expressed Genes.

Front. Neurosci. 12:1026.

doi: 10.3389/fnins.2018.01026

\section{Relationship Between Alzheimer's Disease and the Immune System: A Meta-Analysis of Differentially Expressed Genes}

\author{
Nan Wang ${ }^{1+}$, Ying Zhang ${ }^{2+}$, Li Xu ${ }^{3 \dagger}$ and Shuilin Jin ${ }^{1 *}$ \\ 1 Department of Mathematics, Harbin Institute of Technology, Harbin, China, ${ }^{2}$ Department of Pharmacy, Heilongjiang \\ Province Land Reclamation Headquarters General Hospital, Harbin, China, ${ }^{3}$ College of Computer Science and Technology, \\ Harbin Engineering University, Harbin, China
}

Alzheimer's disease (AD), a neurodegenerative diseases (neuro-diseases) which is prevalent in the elderly and seriously affects the lives of individuals. Many studies have discussed the relationship between immune system and AD pathogenesis. Here, the meta-analysis of differentially expressed (DE) genes based on microarray data was conducted to study the association between AD and immune system. 9519 target genes of hippocampus in 146 subjects ( 73 AD cases and 73 controls) from 4 microarray data sets were compiled and DE genes with $p<1.00 E-04$ were selected to conduct the pathway-analysis. The results indicated that the DE genes were significantly enriched in the neuro-diseases as well as the immune system pathways.

Keywords: Alzheimer's disease, hippocampus, immune pathways, meta-analysis, pathway analysis

\section{INTRODUCTION}

Alzheimer's disease (AD), the common shared and complicated neurodegenerative disorder, is characterized as functional impairment, progressive cognitive dysfunction, and memory loss in the elderly (Pedersen, 2010; Hu et al., 2016). It is likely caused by a series of complex interactions of environmental, lifestyle and genetic factors. About $70 \%$ of the risk is believed to be genetic (Girard et al., 2018). However, the specific genes that contribute to $\mathrm{AD}$ are largely unknown and a great deal of effort has been put into detecting the genetic determinants of AD. There are many methods to investigate the pathogenesis of AD (Cheng et al., 2016a; Yang et al., 2016; Liang et al., 2018) and we used meta-analysis of microarray data to explore the differentially expressed (DE) genes associated with AD (Evangelou and Ioannidis, 2013). Since 2009-2017, large-scale AD studies reported the related impact of genes such as CLU, BIN1, CR1, MS4A6E/MS4A4, PICALM, EPHA1, CD2AP, TREM2, DRB1/ HLA-DRB5, SORL1, SLC24A4-0RING3, PTK2B, MEF2C, DSG2, INPP5D, NME8, FERMT2, CELF1, GAB2, CASS4, and ZCWPW1 (Jiang et al., 2017; Hu et al., 2017a). Recent studies discovered $\mathrm{AD}$ was related to genes including WWC1, ABCA7, APOE, CD33 TRIM22, FOXO3, PP4R3A, DAPK1 (Christopher et al., 2017; Cheng et al., 2018b; Chung, 2018; Gusareva et al., 2018; Moreno-Grau et al., 2018). However, some genes like IGF1 and IGFBP3 may not be contacted with AD (Williams et al., 2017; Hu et al., 2017b, 2018).

Amyloid hypothesis and neurofibrillary tangles have been considered as the most important pathogenesis of $\mathrm{AD}$ in the past decades (Heppner et al., 2015; Cheng et al., 2016b). The sequences of $\mathrm{AD}$ based on amyloid hypothesis are the disposition of the astrogliosis and amyloid$\beta$ peptide, then the presence of neurofibrillary tangles which are largely composed of tau fragments and hyperphosphorylated tau protein, and eventually the loss of neuronal and synaptic function. Inflammatory response is also a character of $\mathrm{AD}$ and escalates with disease progression 
(Hardy and Selkoe, 2002). The relationship between immune system and $\mathrm{AD}$ has not attracted much attention in until recently. However, new research suggests that the immune system, a host defense system, is related with the pathogenesis of $\mathrm{AD}$ (Blasko and Grubeck-Loebenstein, 2003; Cheng et al., 2017). The importance of immune system pathways for the pathogenesis of $\mathrm{AD}$ is highlighted by the results of this article.

We noted that previous studies of microarray datasets applied DE genes with different thresholds of significance including a $q$ value (false discovery rate) with threshold of 0.2 (Rhodes et al., 2002), $p$-value with threshold of 0.01 (Bhargava et al., 2013) and so on. However, the different threshold value chosen for DE genes can reflect the different disease models (Dumur et al., 2004; O'dushlaine et al., 2009). Thus, a significant pathway using DE genes with a smaller threshold might reflect a role for less DE genes of larger effects and a significant pathway enriched by DE genes with a larger threshold may reflect the role of more DE genes with smaller effects (O'dushlaine et al., 2009; Cheng et al., 2018a). In this article, the DE genes with the cut-off of $p$-value $1.00 E-04$ were selected to perform the pathway analysis for exploring the relationship between immune system-mediated actions and Alzheimer's disease.

\section{MATERIALS AND METHODS}

\section{Data}

The microarray database, Gene Expression Omnibus database (GEO database) $^{1}$ (Davis and Meltzer, 2007), was searched for datasets related to AD. Focusing our search on hippocampus brain of human as objects, we identified four publicly available microarray datasets of AD. The gene expression data and detail information about the collecting datasets were available through GEO accession numbers GSE1297 (Blalock et al., 2004), GSE5281 (Liang et al., 2007, 2008; Readhead et al., 2018), GSE28146 (Blalock et al., 2011), and GSE48350 (Berchtold et al., 2008, 2013; Astarita et al., 2010; Cribbs et al., 2012; Sarvari et al., 2012; Blair et al., 2013). Some details about the four microarray datasets of $\mathrm{AD}$ are given below.

Dataset GSE1297 was contributed by Blalock et al. (2004). The investigation explored gene expression data on hippocampal brain of nine controls, $22 \mathrm{AD}$ cases and 31 subjects in total. The participants of this microarray dataset were aged from 65 to 110 years with median age 85 years. The platform of GSE1297 was Affymetrix Human Genome U133A Array (GPL96). For the aim of our investigation we used the data of all 31 samples.

Dataset GSE5281 was provided by Liang et al. (2006). It collected 161 individual brain specimens from three AD Centers including the Washington University Alzheimer's Disease Centers, the Duke University Alzheimer's Disease Centers and the Arizona Alzheimer's Disease Centers. The participants of this microarray dataset were aged from 61 to 101 years with median age 79 years. The platform of GSE5281 was Affymetrix Human Genome U133A Array (GPL570). The study contained gene expression levels on six brain regions including hippocampus,

\footnotetext{
${ }^{1}$ https://www.ncbi.nlm.nih.gov/geo/
}

posterior cingulate, entorhinal cortex, primary visual cortex, medial temporal gyrus, and superior frontal gyrus. For the aim of our study, we used only data on the brain regions of the hippocampus. The GSE5281 contained 23 samples on hippocampal brain, of which 10 samples were AD cases and 13 samples were controls.

Dataset GSE28146 was provided by Blalock et al. (2011) for the aim of exploring the association between $\mathrm{AD}$ and the distinction of gray and white signatures on hippocampus. It collected 30 individual brain specimens including eight controls and $22 \mathrm{AD}$ cases. The participants of this microarray dataset were aged from 65 to 101 years with median age 86 years. The platform of GSE28146 was Affymetrix U133 Plus 2.0 array (GPL570). We use the dataset of 30 samples in total.

Dataset GSE48350 was provided by Berchtold et al. (2014). The datasets contained gene expression levels on four brain regions including entorhinal cortex, hippocampus, post-central gyrus, superior frontal cortex. The participants of this microarray dataset of $\mathrm{AD}$ were aged from 20 to 97 years with median age 69 years. The platform of GSE48350 is Affymetrix U133 Plus 2.0 array (GPL570). For the aim of our analysis, we used only data on the brain regions of the hippocampus. Detailed information about the four datasets is presented in Table $\mathbf{1}$.

We downloaded the series matrix files of the four different microarray expression profilings and the four datasets on $\mathrm{AD}$ were pre-processed by the authors. We applied $\log 2$ transform for the datasets. R packages including hgu133a.db (Carlson, 2016a) and hgu133plus2.db (Gautier et al., 2004; Carlson, 2016b) were used to annotate the datasets. The expression data of the probe sets corresponding to more than one gene were deleted. If a gene was mapped by more than one probe set, the average value of these probe sets was calculated. Then the function MetaDE.merge of MetaDE package (Gentleman et al., 2004; Wang et al., 2012) was used to extract the same genes from all studies. After that, the function MetaDE.filter of MetaDE package was used to filter out genes with very low gene expression level or small variation (cutoff $=0.10)$. Finally, the meta-analysis was conducted on 9519 target genes in 146 samples (73 $\mathrm{AD}$ cases and 73 controls) to explore the relationship between immune system and $\mathrm{AD}$.

\section{Meta-Analysis}

Adaptively weighted Fisher's method was used to detect the DE genes with $p<1.00 E-04$. Adaptively weighted Fisher's method

TABLE 1 | Patient and sample characteristics of Alzhemer's gene expression datasets.

\begin{tabular}{lcccc}
\hline Characteristics & GSE1297 & GSE5281 & GSE28146 & GSE48350 \\
\hline No. of participants & 31 & 23 & 30 & 62 \\
Group, AD:Control & $22: 09$ & $10: 13$ & $22: 08$ & $19: 43$ \\
Median age, ye & $85(65-110)$ & $79(62-101)$ & $86(65-101)$ & $69(20-97)$ \\
$\begin{array}{l}\text { ars (range) } \\
\text { Gender, M:F }\end{array}$ & $13: 18$ & $16: 07$ & $12: 18$ & $32: 30$ \\
$\begin{array}{l}\text { Platforms } \\
\text { GPL96 }\end{array}$ & GPL570 & GPL570 & GPL570 \\
$\begin{array}{l}\text { Genome U133 Plus 2.0 array. } \\
\text { gentrix human genome }\end{array}$ & U133A array; & GPL570, affymetrix human
\end{tabular}


assigned different weight to each individual study:

$$
U_{g}\left(\omega_{g}\right)=-\sum_{i=1}^{k} \omega_{i g} \ln \left(p_{i g}\right), \omega_{i}=0 \text { or } 1
$$

where $i$ represents the $i$ th genetic $\operatorname{study}(i=1,2, \cdots, k), k$ represents the number of microarray studies, $g$ represents the gene $g$, the $p$-value in the $i$ th genetic study of gene $g$ is represented by ${ }^{p_{i g}}$, and assuming $p_{i g} \sim \operatorname{unif}(0,1), \omega_{\text {ig }}$ represents the specific weight assigned to the $i$ th microarray study of gene $g$ Thus, $U_{g}\left(\omega_{g}\right) \sim \operatorname{Gamma}\left(\sum_{i=1}^{k} \omega_{i g}, 1\right)$. Assuming $\omega_{g}=$ $\left(\omega_{1 g}, \omega_{2 g}, \cdots, \omega_{k g}\right)$ and $W=\left[\omega_{g} \mid \omega_{i g}=0\right.$ or 1$]$, AW method searches ${ }^{\omega} g$ through $W$ to find the best weights that provide the minimum final $p$-value.

\section{Pathway-Based Test}

A pathway analysis was conducted based on the DE genes with thresholds of $1.00 E-04$ using a pathway analysis web tool, the Web-based Gene Set Analysis Toolkit provided by Zhang et al. (2005). WebGestalt performs enrichment analysis by incorporating available functional and biological data (Zhang et al., 2005). Here, the Kyoto Encyclopedia of Genes and Genomes (KEGG) pathway in WebGestalt was selected to perform pathway analysis.

\section{RESULTS}

The number of DE genes with $p<1.00 E-04$ detected by AW method was 586 (Supplementary Table 1). The top $10 \mathrm{DE}$ gene KEGG pathways are listed in Table 2.

The significant pathways were associated with some specific diseases including Huntington's disease, $\mathrm{AD}$ and Parkinson's disease. And these enriched pathways were connected mainly with the immune system, like Epithelial cell signaling in Helicobacter pylori infection pathway, Vibrio cholerae infection pathway, Pathogenic Escherichia coli infection pathway, and Phagosome pathway. The results of the meta-analysis suggested that $\mathrm{AD}$ is connected with the immune system.

\section{DISCUSSION}

The differentially expressed genes with threshold of $p<1.00 E-04$ in the meta-analysis were reported. A pathway analysis in KEGG of the DE genes detected by AW method was conducted and the top 10 significant KEGG pathways were reported. Most of pathways were connected with neuro-diseases and the immune system. Here, we further compared our investigation based on the meta-analysis of microarray datasets with other studies.

Stopa et al. (2018) analyzed six healthy controls and seven patients of advanced $\mathrm{AD}$ using transcriptome-wide Affymetrix microarrays, and they reported immune system and metabolic pathways such as cytokine, interferons, cell adhesion, JAK-STAT, acute phase response, and mTOR pathway. However, Cribbs et al. reported that the extent of immune gene upregulation in $\mathrm{AD}$ was modest to the robust response apparent in the aged brain (Cribbs et al., 2012).

Using the DE genes with $p<1.00 E-04$ of meta-analysis methods, we reported significantly enriched top 10 KEGG pathways and discovered that the pathways were associated with neuro-diseases like Huntington's disease pathway (hsa05016), Parkinson's disease pathway (hsa05012), and Synaptic vesicle cycle pathway (hsa04721). Furthermore, most DE genes identified by AW methods were enriched in the immune system including Epithelial cell signaling in H. pylori infection (hsa05120), Pathogenic E. coli infection (hsa05130), V. cholerae infection (hsa05110), and Phagosome (hsa04145).

The infection of $H$. pylori associated with $\mathrm{AD}$ was investigated using histology for diagnosis (Kountouras et al., 2006). The study showed that the pathophysiology of $\mathrm{AD}$ was influenced by $H$. pylori infection through one of the following mechanisms: (1) H. pylori may produce reactive oxygen metabolites and lipid peroxides which accelerate the occurrence of $\mathrm{AD}$ (Malaguarnera et al., 2004). (2) Increasing platelet-leukocyte aggregation and platelet reactivity (Kountouras et al., 2002). Platelets are

TABLE 2 | Significant pathways by pathway analysis of differentially expressed (DE) genes detected by AW method in KEGG.

\begin{tabular}{|c|c|c|c|c|c|c|c|}
\hline PathwayID & Pathway name & C & 0 & E & $\mathbf{R}$ & $P$-Value & FDR \\
\hline hsa00190 & Oxidative phosphorylation & 133 & 24 & 5.13 & 4.67 & $1.69 \mathrm{E}-10$ & 2.57E-08 \\
\hline hsa03050 & Proteasome & 44 & 12 & 1.69 & 7.06 & $6.04 \mathrm{E}-08$ & 4.57E-06 \\
\hline hsa05120 & $\begin{array}{l}\text { Epithelial cell signaling in } \\
\text { Helicobacter pylori infection }\end{array}$ & 68 & 14 & 2.62 & 5.33 & $2.22 \mathrm{E}-07$ & 1.35E-05 \\
\hline hsa05010 & Alzheimer's disease & 171 & 21 & 6.60 & 3.18 & $2.25 \mathrm{E}-06$ & $9.72 \mathrm{E}-05$ \\
\hline hsa05130 & $\begin{array}{l}\text { Pathogenic Escherichia coli } \\
\text { infection }\end{array}$ & 55 & 11 & 2.12 & 5.18 & $6.13 \mathrm{E}-06$ & 0.000232 \\
\hline hsa05110 & Vibrio cholerae infection & 51 & 10 & 1.96 & 5.07 & $1.96 \mathrm{E}-05$ & 0.000661 \\
\hline hsa04145 & Phagosome & 154 & 17 & 5.94 & 2.85 & 8.43E-05 & 0.002554 \\
\hline
\end{tabular}

The pathways are ordered by their $p$-values. $C$, represents the number of reference genes in the category; $O$, represents the number of genes in the gene set and also in the category; $E$, represents expected number in the category; $R$, represents the ratio of enrichment. 
a key component of amyloid which contributes to $\mathrm{AD}$ and causes the occurrence of senile plaque (Kountouras et al., 2006). (3) The cell apoptotic process might be influenced by H. pylori and the cell death has a close relationship with neurodegenerative diseases (neuro-diseases) such as AD (D’Andrea, 2005). (4) $H$. pylori may release a large amount of vasoactive substances and proinflammatory, such as eicosanoids, cytokines and acute phase proteins connected with a sea of disorders of the nervous system including AD (Kountouras et al., 2002). (5) H. pylori might contribute to down syndrome that drives the early onset of the neuro-diseases such as AD (Hallam et al., 2000). E. coli has been found to be closely associated with $\mathrm{AD}$, and E. coli LT and LT (R192G) have been used as mucosal adjuvants to treat AD in mice (Lemere et al., 2002). Rheumatoid arthritis is closely related to $\mathrm{AD}$ and anti-inflammatory agents might be beneficial for $\mathrm{AD}$ (McGeer et al., 1996). In this article, we discovered that AD may be related to $H$. pylori infection, E. coli infection, Rheumatoid arthritis through pathway analysis of KEGG. Moreover, we also found that $\mathrm{AD}$ may be related to $V$. cholerae infection.

In summary, AW meta-analysis method was used to detect the DE genes with strict threshold of $p<1.00 E-04$. The study reported the top ten significantly enriched pathways of the DE genes detected by AW method and our results show that these DE genes are significantly enriched in immune pathways.

\section{AVAILABILITY OF MATERIALS AND DATA}

All data used in this paper are fully publicly available without any restriction.

\section{REFERENCES}

Astarita, G., Jung, K. M., Berchtold, N. C., Nguyen, V. Q., Gillen, D. L., Head, E., et al. (2010). Deficient liver biosynthesis of docosahexaenoic acid correlates with cognitive impairment in Alzheimer's disease. PLoS One 5:e12538. doi: 10.1371/journal.pone.0012538

Berchtold, N. C., Coleman, P. D., Cribbs, D. H., Rogers, J., Gillen, D. L. and Cotman, C. W. (2013). Synaptic genes are extensively downregulated across multiple brain regions in normal human aging and Alzheimer's disease. Neurobiol. Aging 34, 1653-1661. doi: 10.1016/j.neurobiolaging.2012. 11.024

Berchtold, N. C., Cribbs, D. H., Coleman, P. D., Rogers, J., Head, E., Kim, R., et al. (2008). Gene expression changes in the course of normal brain aging are sexually dimorphic. Proc. Natl. Acad. Sci. U.S.A. 105, 15605-15610. doi: 10.1073/pnas.0806883105

Berchtold, N. C., Sabbagh, M. N., Beach, T. G., Kim, R. C., Cribbs, D. H., and Cotman, C. W. (2014). Brain gene expression patterns differentiate mild cognitive impairment from normal aged and Alzheimer's disease. Neurobiol. Aging. 35, 1961-1972. doi: 10.1016/j.neurobiolaging.2014.03.031

Bhargava, A., Clabaugh, I., To, J. P., Maxwell, B. B., Chiang, Y. H., Schaller, E. G., et al. (2013). Identification of cytokinin responsive genes using microarray meta-analysis and RNA-seq in Arabidopsis thaliana. Plant. Physiol. 113:217026. doi: 10.1104/pp.113.217026

Blair, L. J., Nordhues, B. A., Hill, S. E., Scaglione, K. M., O’Leary, J. C., Fontaine, S. N., et al. (2013). Accelerated neurodegeneration through chaperonemediated oligomerization of tau. J. Clin. Invest. 123, 4158-4169. doi: 10.1172/ JCI69003

Blalock, E. M., Buechel, H. M., Popovic, J., Geddes, J. W., and Landfield, P. W. (2011). Microarray analyses of laser-captured hippocampus reveal distinct gray
Dataset GSE1297 is available from the link of https://www. ncbi.nlm.nih.gov/geo/query/acc.cgi?acc=GSE1297.

Dataset GSE5281 can be got from the URL of https://www. ncbi.nlm.nih.gov/geo/query/acc.cgi?acc=GSE5281.

Dataset GSE28146 is available at https://www.ncbi.nlm.nih. gov/geo/query/acc.cgi?acc=GSE28146.

Dataset GSE48350 can be got from the URL: https://www.ncbi. nlm.nih.gov/geo/query/acc.cgi?acc=GSE48350.

\section{AUTHOR CONTRIBUTIONS}

SJ provided the guidance during the whole research. NW collected the data. LX carried out data analysis. YZ and LX wrote the manuscript.

\section{FUNDING}

This project was supported by grants from the China Natural Science Foundation (Grant No. 11301110), Natural Science Foundation of Heilongjiang Postdoctoral Fund (Grant No. LBH-Q18049).

\section{SUPPLEMENTARY MATERIAL}

The Supplementary Material for this article can be found online at: https://www.frontiersin.org/articles/10.3389/fnins. 2018.01026/full\#supplementary-material

and white matter signatures associated with incipient Alzheimer's disease. J. Chem. Neuroanat. 42, 118-126. doi: 10.1016/j.jchemneu.2011.06.007

Blalock, E. M., Geddes, J. W., Chen, K. C., Porter, N. M., Markesbery, W. R., and Landfield, P. W. (2004). Incipient Alzheimer's disease: microarray correlation analyses reveal major transcriptional and tumor suppressor responses. Proc. Natl. Acad. Sci. U.S.A. 101, 2173-2178. doi: 10.1073/pnas.0308512100

Blasko, I., and Grubeck-Loebenstein, B. (2003). Role of the immune system in the pathogenesis, prevention and treatment of Alzheimer's disease. Drugs Aging 20, 101-113. doi: 10.2165/00002512-200320020-00002

Carlson, M. (2016a). hgu133a.db: Affymetrix Human Genome U133 Set annotation data (chip hgu133a). R package version. 3.2.3.

Carlson, M. (2016b). hgu133plus2.db: Affymetrix Human Genome U133 Plus 2.0 Array annotation data (chip hgu133plus2). R package version. 3.2.3.

Cheng, L., Hu, Y., Sun, J., Zhou, M., and Jiang, Q. (2018a). DincRNA: a comprehensive web-based bioinformatics toolkit for exploring disease associations and ncRNA function. Bioinformatics 34, 1953-1956. doi: 10.1093/ bioinformatics/bty002

Cheng, L., Jiang, Y., Ju, H., Sun, J., Peng, J., Zhou, M., et al. (2018b). InfAcrOnt: calculating cross-ontology term similarities using information flow by a random walk. BMC Genomics 19:919. doi: 10.1186/s12864-017-4338-6

Cheng, L., Jiang, Y., Wang, Z., Shi, H., Sun, J., Yang, H., et al. (2016a). DisSim: an online system for exploring significant similar diseases and exhibiting potential therapeutic drugs. Sci. Rep. 6:30024. doi: 10.1038/srep30024

Cheng, L., Sun, J., Xu, W., Dong, L., Hu, Y., and Zhou, M. (2016b). OAHG: an integrated resource for annotating human genes with multi-level ontologies. Sci. Rep. 10:34820. doi: 10.1038/srep34820

Cheng, L., Yang, H., Zhao, H., Pei, X., Shi, H., Sun, J., et al. (2017). MetSigDis: a manually curated resource for the metabolic signatures of diseases. Brief Bioinform. doi: 10.1093/bib/bbx103 [Epub ahead of print]. 
Christopher, L., Napolioni, V., Khan, R. R., Han, S. S., Greicius, M. D., and Initiative, A. D. N. (2017). A variant in ppp4r3a protects against alzheimerrelated metabolic decline. Ann. Neurol. 82, 900-911. doi: 10.1002/ana. 25094

Chung, J. (2018). Genetic Association Studies of Alzheimer Disease Using MultiPhenotype Tests and Gene-Based Tests. Available at: https://hdl.handle.net/2144/ 27693.

Cribbs, D. H., Berchtold, N. C., Perreau, V., Coleman, P. D., Rogers, J., Tenner, A. J., et al. (2012). Extensive innate immune gene activation accompanies brain aging, increasing vulnerability to cognitive decline and neurodegeneration: a microarray study. J. Neuroinflammation 9:179. doi: 10.1186/1742-20949-179

D'Andrea, M. R. (2005). Add Alzheimer's disease to the list of autoimmune diseases. Med. Hypotheses 64, 458-463. doi: 10.1016/j.mehy.2004. 08.024

Davis, S., and Meltzer, P. S. (2007). GEOquery: a bridge between the Gene Expression Omnibus (GEO) and BioConductor. Bioinformatics 23, 1846-1847. doi: 10.1093/bioinformatics/btm254

Dumur, C. I., Nasim, S., Best, A. M., Archer, K. J., Ladd, A. C., Mas, V. R., et al. (2004). Evaluation of quality-control criteria for microarray gene expression analysis. Clin. Chem. 50, 1994-2002. doi: 10.1373/clinchem.2004. 033225

Evangelou, E., and Ioannidis, J. P. (2013). Meta-analysis methods for genome-wide association studies and beyond. Nat. Rev. Genet. 14, 379-389. doi: 10.1038/ $\operatorname{nrg} 3472$

Gautier, L., Cope, L., Bolstad, B. M., and Irizarry, R. A. (2004). Affy-analysis of Affymetrix GeneChip data at the probe level. Bioinformatics 20, 307-315. doi: 10.1093/bioinformatics/btg405

Gentleman, R. C., Carey, V. J., Bates, D. M., Bolstad, B., Dettling, M., Dudoit, S., et al. (2004). Bioconductor: open software development for computational biology and bioinformatics. Genome. Biol. 5:R80.

Girard, H., Potvin, O., Nugent, S., Dallaire-Théroux, C., Cunnane, S., Duchesne, S., et al. (2018). Faster progression from MCI to probable $\mathrm{AD}$ for carriers of a single-nucleotide polymorphism associated with type 2 diabetes. Neurobiol. Aging 64, 157.e11-157.e17. doi: 10.1016/j.neurobiolaging.2017. 11.013

Gusareva, E. S., Twizere, J. C., Sleegers, K., Dourlen, P., Abisambra, J. F., Meier, S., et al. (2018). Male-specific epistasis between WWC1 and TLN2 genes is associated with Alzheimer's dissease. Neurobiol. Aging 72, 188.e3-188.e12. doi: 10.1016/j.neurobiolaging.2018.08.001

Hallam, D., Capps, N., Travelstead, A., Brewer, G., and Maroun, L. (2000). Evidence for an interferon-related inflammatory reaction in the trisomy 16 mouse brain leading to caspase-1-mediated neuronal apoptosis. J. Neuroimmunol. 110, 66-75. doi: 10.1016/S0165-5728(00)00289-7

Hardy, J., and Selkoe, D. J. (2002). The amyloid hypothesis of Alzheimer's disease: progress and problems on the road to therapeutics. Science 297, 353-356. doi: 10.1126/science.1072994

Heppner, F. L., Ransohoff, R. M., and Becher, B. (2015). Immune attack: the role of inflammation in Alzheimer disease. Nat. Rev. Neurosci. 16, 358-372. doi: 10.1038/nrn3880

Hu, Y., Cheng, L., Zhang, Y., Bai, W., Wang, T., Han, Z., et al. (2017a). Rs4878104 contributes to Alzheimer's disease risk and regulates DAPK1 gene expression. Neurol. Sci. 38, 1255-1262. doi: 10.1007/s10072-0172959-9

Hu, Y., Zheng, L., Cheng, L., Zhang, Y., Bai, W., Zhou, W., et al. (2017b). GAB2 rs2373115 variant contributes to Alzheimer's disease risk specifically in European population. J. Neurol. Sci. 375, 18-22. doi: 10.1016/j.jns.2017. 01.030

Hu, Y., Zhao, T., Zhang, N., Zang, T., Zhang, J., and Cheng, L. (2018). Identifying diseases-related metabolites using random walk. BMC Bioinformatics 19:116. doi: 10.1186/s12859-018-2098-1

Hu, Y., Zhou, M., Shi, H., Ju, H., Jiang, Q., and Cheng, L. (2016). Measuring disease similarity and predicting disease-related ncRNAs by a novel method. BMC. Med. Genomics. 10:71. doi: 10.1186/s12920-017-0315-9

Jiang, Q., Jin, S., Jiang, Y., Liao, M., Feng, R., Zhang, L., et al. (2017). Alzheimer's disease variants with the genome-wide significance are significantly enriched in immune pathways and active in immune cells. Mol. Neurobiol. 54, 594-600. doi: $10.1007 /$ s12035-015-9670-8
Kountouras, J., Mylopoulos, N., Chatzopoulos, D., Zavos, C., Boura, P., Konstas, A. G., et al. (2002). Eradication of Helicobacter pylori may be beneficial in the management of chronic open-angle glaucoma. Arch. Intern. Med. 162, 1237-1244. doi: 10.1001/archinte.162.11.1237

Kountouras, J., Tsolaki, M., Gavalas, E., Boziki, M., Zavos, C., Karatzoglou, P., et al. (2006). Relationship between Helicobacter pylori infection and Alzheimer disease. Neurology 66, 938-940. doi: 10.1212/01.wnl.0000203644.68 $059.5 \mathrm{f}$

Lemere, C. A., Spooner, E. T., Leverone, J. F., Mori, C., and Clements, J. D. (2002). Intranasal immunotherapy for the treatment of Alzheimer's disease: Escherichia coli LT and LT (R192G) as mucosal adjuvants. Neurobiol. Aging 23, 991-1000. doi: 10.1016/S0197-4580(02)00127-6

Liang, W. S., Dunckley, T., Beach, T. G., Grover, A., Mastroeni, D., Walker, D. G., et al. (2007). Gene expression profiles in anatomically and functionally distinct regions of the normal aged human brain. Physiol. Genomics 28, 311-322. doi: 10.1152/physiolgenomics.00208.2006

Liang, W. S., Reiman, E. M., Valla, J., Dunckley, T., Beach, T. G., Grover, A., et al. (2008). Alzheimer's disease is associated with reduced expression of energy metabolism genes in posterior cingulate neurons. Proc. Natl. Acad. Sci. U.S.A. 105, 4441-4446. doi: 10.1073/pnas.0709259105

Liang, X., Schnetz-Boutaud, N., Kenealy, S. J., Jiang, L., Bartlett, J., Lynch, B., et al. (2006). Covariate analysis of late-onset Alzheimer disease refines the chromosome 12 locus. Mol. Psychiatry 11, 280-285. doi: 10.1038/sj.mp.4001766

Liang, Z., Morisseau, C., Hwang, S. H., Hammock, B. D., and Li, Q. X. (2018). A dual-inhibitor of soluble epoxide hydrolase and p38 kinase alleviating tau hyperphosphorylation and amyloid neurotoxicity for potential treatment of neuroinflammation in Alzheimer's disease. Faseb. J. 32:559. doi: 10.1096/fasebj. 2018.32.1_supplement.559.2

Malaguarnera, M., Bella, R., Alagona, G., Ferri, R., Carnemolla, A., and Pennisi, G. (2004). Helicobacter pylori and Alzheimer's disease: a possible link. Eur. J. Intern. Med. 15, 381-386. doi: 10.1016/j.ejim.2004.05.008

McGeer, P. L., Schulzer, M., and McGeer, E. G. (1996). Arthritis and antiinflammatory agents as possible protective factors for Alzheimer's disease A review of 17 epidemiologic studies. Neurology 47, 425-432. doi: 10.1212/WNL. 47.2.425

Moreno-Grau, S., Hernández, I., Heilmann-Heimbach, S., Ruiz, S., RosendeRoca, M., Mauleón, A., et al. (2018). Genome-wide significant risk factors on chromosome 19 and the APOE locus. Oncotarget 9, 24590-24600. doi: 10. 18632/oncotarget. 25083

O’dushlaine, C., Kenny, E., Heron, E. A., Segurado, R., Gill, M., Morris, D. W., et al. (2009). The SNP ratio test: pathway analysis of genome-wide association datasets. Bioinformatics. 25, 2762-2763. doi: 10.1093/bioinformatics/ btp 448

Pedersen, N. L. (2010). Reaching the limits of genome-wide significance in Alzheimer's disease: back to the environment. JAMA 303, 1864-1865. doi: 10. 1001/jama.2010.609

Readhead, B., Haure-Mirande, J. V., Funk, C. C., Richards, M. A., Shannon, P. Haroutunian, V., et al. (2018). Multiscale analysis of independent alzheimer's cohorts finds disruption of molecular, genetic, and clinical networks by human herpesvirus. Neuron 99, 64.e7-82.e7. doi: 10.1016/j.neuron.2018.05.023

Rhodes, D. R., Barrette, T. R., Rubin, M. A., Ghosh, D., and Chinnaiyan, A. M. (2002). Meta-analysis of microarrays: interstudy validation of gene expression profiles reveals pathway dysregulation in prostate cancer. Cancer Res. 62, 4427-4433.

Sarvari, M., Hrabovszky, E., Kallo, I., Solymosi, N., Liko, I., Berchtold, N., et al. (2012). Menopause leads to elevated expression of macrophageassociated genes in the aging frontal cortex: rat and human studies identify strikingly similar changes. J. Neuroinflammation 9:264. doi: 10.1186/17422094-9-264

Stopa, E. G., Tanis, K. Q., Miller, M. C., Nikonova, E. V., Podtelezhnikov, A. A., Finney, E. M., et al. (2018). Comparative transcriptomics of choroid plexus in Alzheimer's disease, frontotemporal dementia and Huntington's disease: implications for CSF homeostasis. Fluids Barriers CNS 15:18. doi: 10.1186/ s12987-018-0102-9

Wang, X., Kang, D. D., Shen, K., Song, C., Lu, S., Chang, L. C., et al. (2012). An $\mathrm{R}$ package suite for microarray meta-analysis in quality control, differentially expressed gene analysis and pathway enrichment detection. Bioinformatics 28, 2534-2536. doi: 10.1093/bioinformatics/bts485 
Williams, D. M., Karlsson, I. K., Pedersen, N. L., and Hägg, S. (2017). Circulating insulin-like growth factors and Alzheimer disease: a meta-analysis. Neurology 10:1212.

Yang, G., Wang, Y., Sun, J., et al. (2016). Ginkgo biloba for mild cognitive impairment and alzheimer's disease: a systematic review and meta-analysis of randomized controlled trial. Curr. Top. Med. Chem. 16, 520-528. doi: 10.2174/ 1568026615666150813143520

Zhang, B., Kirov, S., and Snoddy, J. (2005). WebGestalt: an integrated system for exploring gene sets in various biological contexts. Nucleic Acids Res. 33, W741-W748. doi: 10.1093/nar/gki475
Conflict of Interest Statement: The authors declare that the research was conducted in the absence of any commercial or financial relationships that could be construed as a potential conflict of interest.

Copyright $\odot 2019$ Wang, Zhang, Xu and Jin. This is an open-access article distributed under the terms of the Creative Commons Attribution License (CC BY). The use, distribution or reproduction in other forums is permitted, provided the original author(s) and the copyright owner(s) are credited and that the original publication in this journal is cited, in accordance with accepted academic practice. No use, distribution or reproduction is permitted which does not comply with these terms. 